\title{
THE DESIGN METHOD OF ROBUST CONTROL BY FLEXIBLE SPACECRAFT
}

\author{
V.Yu. Rutkovsky, S.D. Zemlyakov, V.M. Sukhanov, V.M. Glumov \\ Institute of Control Sciences, Russian Academy of Sciences, \\ Profsoyuznaya 65, 117997, Moscow, Russia \\ Tel:+7(095)334 87 30; e-mail : rutkov@ipu.rssi.ru
}

\begin{abstract}
The problem of angular motion stabilization by mechanical systems with nonrigid construction is considered. The control is realized in the class of discrete systems with piecewise constant control actions which can lead to appearance and growth of the elastic oscillations of the object construction. The notion of the influence function of a base control on the elastic oscillations is introduced. Using this function the task of the subsystem formation of the intellectual diagnostics and tuning of the base algorithm is solved. Some results of digital simulation of the suggested method of control by multifrequency large space structure are given. Copyright (C) 2005 IFAC
\end{abstract}

Keywords: Large space structures, oscillation, robust control

\section{INTRODUCTION AND STATEMENT OF THE PROBLEM}

There are many types of compound technical objects that require orientation of their position into space. The requirements for decrease of the metal expenditure for their manufacture lead to elasticity of the construction. As the result they are flexible multifrequency objects. The typical examples of such objects are the large space structures (LSS) (Nurre, et al., 1985), (Kirk, (ed.), 1990, 1993, 1996, 1999), space and submarine robotic modules that have long manipulators links and long payloads. Such exotic objects as civil earthquake-proof multi-storied buildings on moving foundation belong to this class of the objects too (Spencer and Soong, 1999).

The peculiarities of considered objects are the existence of a main rigid body containing sensors and actuators of the control system. Translational and rotational motions are defined by the coordinates $q^{\mathrm{t}}$ and $q^{\mathrm{r}}$ of the body-fixed frame $O x y z$ in the inertial space. Attached to the main body additional elements or blocks can be flexible. Their positions are defined by finite number $n$ of the generalized coordinates $q_{\text {ad }}$. First of all it must be noted that further the specific peculiarities of control by space vehicles are taking into account. But the most results are also true for all aforementioned objects.

Dynamics of the considered objects usually is described by Lagrange equations that can be reduced to the following form of the finite-elements model (Nurre, et al., 1985)

$$
A(q) \ddot{q}+H \dot{q}+B q=Q+R,
$$

where $q=\left(q_{0}^{\mathrm{T}}, q_{\mathrm{ad}}^{\mathrm{T}}\right)^{\mathrm{T}}$ is the $\left(n^{\prime} \times 1\right)$ vector of generalized coordinates that are defined the position and configuration of the flexible object $\left(n^{\prime}=n+6\right.$, $\left.q_{0}=\left(q^{\mathrm{tT}}, q^{\mathrm{rT}}\right)^{T}\right) ; A, B$ are the symmetric $\left(n^{\prime} \times n^{\prime}\right)$ matrices of the masses and rigidities; $H$ is the ( $n^{\prime} \times n^{\prime}$ ) -matrix of damping; $Q=K(q) \mu$ is the vector of generalized forces; $K(q)$ is the $\left(n^{\prime} \times 6\right)$ matrix of the actuators effectiveness; $\mu=\left(\mu^{\mathrm{tT}}, \mu^{\mathrm{rT}}\right)^{\mathrm{T}}$ is the vector of control actions; $\mu^{\mathrm{t}}=\left(\mu_{1}^{\mathrm{t}}, \mu_{2}^{\mathrm{t}}, \mu_{3}^{\mathrm{t}}\right)^{\mathrm{T}}$ is the subvector of translational motion control and $\mu^{\mathrm{r}}=\left(\mu_{1}^{\mathrm{r}}, \mu_{2}^{\mathrm{r}}, \mu_{3}^{\mathrm{r}}\right)^{\mathrm{T}}$ is the subvector of rotational mo- 
tion control by the main body; $R=R(q, \dot{q})$ is the vector of Coriolis and centrifugal forces. As a rule the components of the $(6 \times 1)$ vector $q_{0}$ are the controlled coordinates. Considered in this paper problem is retention of the vector coordinates $q_{0}$ in the domain of small displacements from their desired values $q_{0}^{0} \equiv 0$. Small displacements of the vector components $q$ allow to use the linearized equation

$$
A^{*} \ddot{x}+H \dot{x}+B x=K^{*} \mu
$$

where $x=\left(x_{0}^{\mathrm{T}}, x_{\mathrm{ad}}^{\mathrm{T}}\right)^{\mathrm{T}}, x_{0}=q_{0}, x_{\mathrm{ad}}=q_{\mathrm{ad}} ; A^{*}, K^{*}$ are constant matrices now.

Very often it is possible to assume that in equation (2) the components of the matrices defining translational and rotational inter-motion coupling are small. It is valid in the motion of the object into space or in the motion underwater at low velocities. In this case the term $H \dot{x}$ is small too. Then equation (2) is divided into two analogous systems that in the first approximation are independent. Each system after some transformations has the following form

$$
\left[\begin{array}{cc}
A_{11} & A_{12} \\
A_{21} & A_{22}
\end{array}\right]\left[\begin{array}{l}
\ddot{x}_{0} \\
\ddot{x}_{\mathrm{ad}}
\end{array}\right]+\left[\begin{array}{ll}
0 & 0 \\
0 & B
\end{array}\right]\left[\begin{array}{c}
x_{0} \\
x_{\mathrm{ad}}
\end{array}\right]=\left[\begin{array}{c}
K \mu \\
0
\end{array}\right],
$$

where $x_{0}=x_{0}^{\mathrm{t}}, x_{\mathrm{ad}}=x_{\mathrm{ad}}^{\mathrm{t}}, \mu=\mu^{\mathrm{t}}$ for translational motion and $x_{0}=x_{0}^{\mathrm{r}}, x_{\mathrm{ad}}=x_{\mathrm{ad}}^{\mathrm{r}}, \mu=\mu^{\mathrm{r}}$ for rotational motion. $A_{i j}=A_{i j}^{\mathrm{t}}$ or $A_{i j}=A_{i j}^{\mathrm{r}}, i, j=1,2$, and $K=K^{\mathrm{t}}$ or $K=K^{\mathrm{r}}$ are the sub-matrices of the matri$\operatorname{ces} A^{*}$ and $K^{*}$.

For definiteness in this paper rotational motion of the object is considered. A solution of the task of the algorithms synthesis of energy economic and robust control by nonrigid objects described by equation (3) is suggested. The requirement to have economic control is defined by limited store of the energy for control under condition of long-duration operation of spacecrafts on the orbit. The requirement to have robust control is defined by the fact that it is impossible to calculate the exact values of the flexible spacecraft parameters under earthly conditions.

\section{TRANSFORMATION OF THE OBJECT'S MODEL TO THE MODAL-PHYSICAL FORM}

As the first step of the suggested method of designing control system is the transformation of the equation (3) to the modal-physical form (Glumov, et al., 1998). For that the coordinates $x_{i}$ of the vector $x$ are represented as the sum of two components

$$
x_{i}=\bar{x}_{i}+\tilde{x}_{i}, \tilde{x}_{i}=\sum_{j=1}^{n} \tilde{x}_{i j}, i=1,2,3
$$

Here $\bar{x}_{i}$ are the coordinates of the main body motion in the case if all construction would be rigid; $\tilde{x}_{i}$ are additional displacements of the coordinates $x_{i}$ due to the elastic oscillations of the attached flexible elements. The coordinates of the vector $x=\left(x_{i}\right), i=1,2,3$, are considered as Euler angles.

Separation (4) of the coordinates $x_{i}$ permits to represent equation (3) in the following form

$$
\begin{gathered}
\ddot{\bar{x}}=N m_{\mu} ; \ddot{s}+\omega^{2} s=G m_{\mu} ; \tilde{x}=L s ; x=\bar{x}+\tilde{x}, \\
x=\bar{x}+\tilde{x},
\end{gathered}
$$

where $s=\left(s_{j}\right)$ is $n \times 1$-vector of normal coordinates; $\omega^{2}=\operatorname{diag}\left(\omega_{j}^{2}\right), j=\overline{1, n} ; \omega_{j}$ are fundamental frequencies of the elastic oscillations of the object; $m_{\mu}=J^{-1} K \mu ; \quad J=\operatorname{diag}\left(J_{11}, J_{22}, J_{33}\right) ; \quad N=A_{11}^{-1} J$; $L$ and $G$ are the matrices defined in (Glumov, et al., 1998).

If gas-jet engines or hand-wheels are used as control devices the system of equations (5) may be divided into three independent modal-physical models (MPM) (Glumov, et al., 1998) each of that represents the object rotation $x_{i}, i=\overline{1,3}$, with respect to any of three orthogonal axes of the inertial coordinate system (further subscript $i$ will be omitted).

In the scalar form MPM is written as follows:

$$
\mathfrak{M}: \ddot{\bar{x}}=m, \quad \ddot{\tilde{x}}_{j}+\omega_{j}^{2} \tilde{x}_{j}=\tilde{k}{ }_{j} m, \quad x=\bar{x}+\tilde{x}, \quad \tilde{x}=\sum_{j=1}^{n} \tilde{x}_{j}
$$

The object output $x=\bar{x}+\tilde{x}$ is measured by angularrate sensor with digital output $z=\bar{z}+\tilde{z}$ where $\bar{z}=\bar{x}[k], \tilde{z}=\tilde{x}[k], k$ is discrete time.

\section{THE CHARACTERISTICS OF THE REGULATORS INFLUENCE ON THE ELASTIC OSCILLATIONS}

As distinct from (Krutova, 2001) let us introduce the notion "model of the regime's set of the dominant mode" instead of the set of autonomous models of the isolated elastic modes. It is defined as MPM $\mathfrak{M}_{d},(d \in j=\overline{1, n})$ in scalar form (7) and it is distinguished by particular combination of the vector components initial conditions values $\tilde{x}^{0}=\left(\tilde{x}_{j}^{0}\right)$, when $\left|\tilde{x}_{d}^{0}\right| \gg\left|\tilde{x}_{j}^{0}\right| \approx 0 \forall j \neq d$ and $\left|\bar{x}^{0}\right|,\left|\dot{\bar{x}}^{0}\right| \geq 0$. This model $\mathfrak{M}_{d}$ for brevity is called as the model of the object's $d$-regime $(d=\overline{1, n})$.

Further the discrete control systems that are widely applied for space objects are considered. In such systems the steady-state motion is represented by a sta- 
ble limit cycle $\Gamma=\Gamma\left[u\left(z, r_{v}\right)\right]$ with period $\tau_{\Gamma}$ and admissible amplitude. The influence of the control action $m\left[u\left(z, r_{v}\right)\right]$ on the oscillating component $\tilde{x}_{d}(t)$ of the model $\mathfrak{M}_{d}$ can be determined with the help of a quasi-envelope $\rho_{d}\left(t, r_{v}\right)=\operatorname{Env}\left[\tilde{x}_{d}\left(u\left(z, r_{v}\right), t\right)\right]$ of the transient process of the component $\tilde{x}_{d}(t)$ on the time interval $\tau_{a}$ (active interval) belonging to the limit cycle period $\tau_{\Gamma}$ when $m[u] \neq 0$. This envelope can be defined by the function

$$
\rho_{d}\left(t, \lambda_{d}\left(r_{v}\right)\right)=a e^{\lambda_{d}\left(r_{v}\right) t} .
$$

In designing control system envelope (8) is calculated without trouble using equations (7). But the frequecies $\omega_{j}$ can not be known exactly and moreover they can change during the active life of the object. So it is necessary to have the method of envelope (8) calculation in the process of the object operation. In this case the following method can be suggested. According to this method a set $Z_{s}=\{\tilde{z}[k]\}$ is used. It can be obtained for example by processing of the coordinate $z[k]$ by the method of Kalman filtration (Ermilova, et al., 2004).

The outputs of the Kalman filter are $\bar{z}[k]$ and $\tilde{z}[k]$. But it is appropriate to obtain the component $\tilde{z}[k]$ as the difference $\tilde{z}[k]=z[k]-\bar{z}[k]$ since the component $\bar{z}[k]$ on the Kalman filter output converges more quickly and more accurately in compare with the component $\tilde{z}[k]$. Moreover the component $\tilde{z}[k]$ on the Kalman filter output contains only some modes of the elastic oscillations. Further the operation of rectification, $z_{a}[k]=|\tilde{z}[k]|$ is used with subsequent selection of all maximum values $z_{m}[l]$. The set $Z_{s}$ is transformed into the set $Z_{m}=\left\{z_{m}[l]\right\}$ jointly with the set of the correspondent instants $T_{m}=\{l\}$. Defined by the sets $Z_{m}, T_{m}$ the function $f=\left\{\left(l, z_{m}\right) \in T_{m} \times Z_{m} \mid z_{m}=f(l)\right\}$ is subjected to two-stage approximation. At the first stage this function is replaced by the approximating polynomial $P(t)=\sum_{v=0}^{q} p_{v}\left(t-l_{1}\right)^{v}$, where $p_{v}$ are constant coefficients, $l_{1}$ is the first element of the set $T_{m}$. Approximation is realized by the method of least squares and function "Polifit" in MATLAB. The second stage is the procedure of minimization of the functional $J_{\lambda}=\int_{\tau_{0}}^{\tau_{a}}\left[P\left(\tau_{0}\right) e^{\lambda_{d}\left(r_{v}\right) \tau}-P(\tau)\right]^{2} d \tau$. Calculated the value $\left|\lambda_{d}\left(r_{v}\right)\right|$ defines the rate of the component $\tilde{x}_{d}$ amplitude changing. The $\operatorname{sign} \lambda_{d}$ defines the character of the oscillating process: at $\lambda_{d}<0$ it converges $\left(\tilde{x}_{d}\left(r_{v}, t\right) \in \tilde{x}_{d}^{-}\right)$; at $\lambda_{d}>0$ it diverges $\left(\tilde{x}_{d}\left(r_{v}, t\right) \in \tilde{x}_{d}^{+}\right)$.
If $\left|\lambda_{d}\left(r_{v}\right)\right| \leq \varepsilon \rightarrow 0$ then for $\operatorname{sign}\left[\lambda_{d}\left(r_{v}\right)\right]= \pm 1$ the regulator affects on the elastic component weakly $\left(\tilde{x}_{d}\left(r_{v}, t\right) \in \tilde{x}_{d}^{0}\right)$. So for any constant value $r_{*}$ of the parameter $r_{v}$ the influence of the regulator on the oscillating component $\tilde{x}_{d}(t)$ of the model $\mathfrak{M}_{d}$ can be defined by the single number $\lambda_{d^{*}}=\lambda_{d}\left(r_{*}\right)$. Varying the parameter $r_{v}$, repeating for any new value $r_{v}$ computer simulation of the object stabilization regime with the model $\mathfrak{M}_{d}$ and calculating the index of the quasi-envelope $\lambda_{d}$ some influence function $\lambda_{d}=\lambda_{d}\left(r_{v}\right)$ will be obtained. This function reflects the influence of the base algorithm $u\left(z, r_{v}\right)$ on the elastic oscillations for $d$-regime. The totality of the influence functions $\Lambda_{d}=\left\{\lambda_{d}\left(r_{v}\right)\right\}(d=\overline{1, n})$ is used further as an informational index for the subsystem of the intellectual diagnosis (Dubrovin, et al., 2003) of the oscillating component condition.

The example of limited number of the influence functions $\lambda_{d}\left(r_{v}\right), d=\overline{1,3}$, for the LSS and discrete analog of PD-algorithm stabilization with a discreteness period $T_{0}$ (Krutova, 2001) (within period $T_{0}$ control action $m=$ const) is shown in Fig. 1. The coefficients of model (7) for $n=6, \quad \mathrm{I}=2500 \mathrm{kgm}^{2}$, $\mathrm{I}_{0}=167 \mathrm{kgm}^{2}$ are indicated in Table 1 .

Table 1.

\begin{tabular}{ccrrrcc}
\hline$\tilde{x}_{i}$ & $i=1$ & $i=2$ & $i=3$ & $i=4$ & $i=5$ & $i=6$ \\
\hline$f_{i}[\mathrm{~Hz}]$ & 1.3 & 1.6 & 1.9 & 2.2 & 2.5 & 3.2 \\
$\omega_{i}$ & 8.17 & 10.05 & 11.94 & 13.82 & 15.7 & 20.1 \\
$\tilde{k}_{i}$ & 8.5 & 5.6 & 4.8 & 1.5 & 0.4 & 0.2 \\
\hline
\end{tabular}

Analysis of the influence functions makes it possible to select two domains of active $\left(r_{v} \equiv T_{0} \leq 1,5 \mathrm{~s}\right)$ and

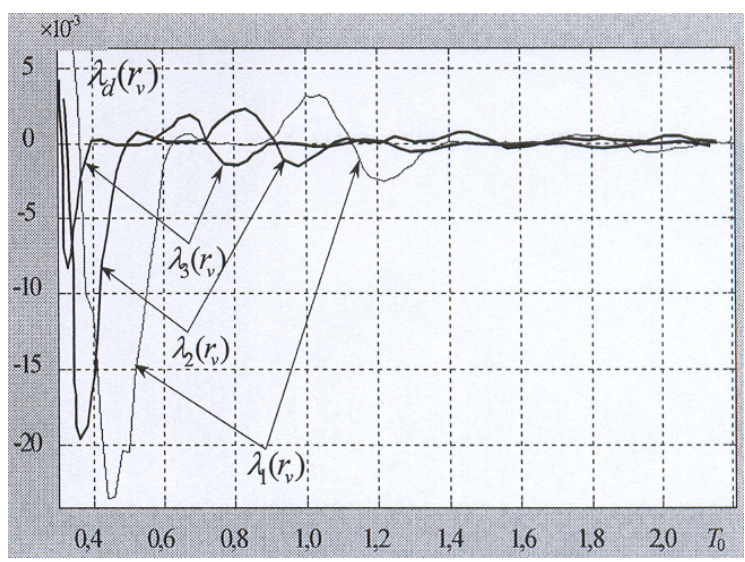

Fig.1. Totality of the influence functions.

neutral $\left(r_{v}>1,5 s\right)$ influence of the regulator on the elastic oscillations. And it is clear in what domain of 
varying parameter $r_{v}$ one of the dominant modes $(d=1,2,3)$ is the cause of the LSS motion instability.

The task of the influence functions $\lambda_{d}\left(r_{v}\right)$ analysis can be considered as a prediction of possible critical regimes of the LSS dynamics (hunting phenomenon of the regulator by the elastic oscillations and others (Rutkovsky and Sukhanov, 1973)). These functions can be used both in designing control system and for realization of robust control by the object (tuning of the parameter $r_{v}$ during the flight). The most interesting task is the last one.

Let in the system under influence of the control action $m\left[u\left(z, r_{0}\right)\right],\left(r_{0} \in\left[r_{\min }, r_{\max }\right]\right.$ is the nominal value of the tuned parameter) $d$-regime occur which is estimated using the set $Z_{m}=\left\{z_{m}[l]\right\}$. It is required:

1. To define the number " $d "$ of the dominant mode on the basis of the frequency $\omega_{d}, d=\overline{1, n}$, identification procedure;

2. Using the number " $d$ " it is necessary to choose from the totality $\Lambda_{d}=\left\{\lambda_{d}\left(r_{v}\right)\right\}$ that are stored in the computer the influence function $\lambda_{d}\left(r_{v}\right)$ and to define new value $r_{1} \in\left[r_{\text {min }}, r_{\text {max }}\right]$ with regard to necessary $\quad\left(\operatorname{sign}\left[\lambda_{d}\left(r_{1}\right)\right]=-1\right) \quad$ and sufficient $\left(\lambda_{d}\left(r_{1}\right)=\left(\lambda_{d}\right)_{\min } \forall r \in\left[r_{1}^{-}, r_{1}^{+}\right]\right)$conditions at which the control action $m\left[u\left(z, r_{1}\right)\right]$ guarantees maximum rate of the dominant mode damping. Here $r_{1}^{-}, r_{1}^{+}$ are the boundaries of the domain in which necessary condition $\left(\lambda_{d}\left(r_{1}\right) \leq 0\right)$ of the component $\tilde{x}_{d}(t)$ damping is fulfilled.

The solution of this task realizes the synthesis procedure of the subsystem of the base algorithm adaptive tuning. The goal of this tuning is the dominant mode damping without additional energy consumption.

\section{DESIGNING SUBSYSTEM OF THE BASE ALGORITHM PARAMETER TUNING}

In the synthesis of the subsystem of the varying parameter $r_{v}$ tuning the sets $Z_{m}$ and $T_{m}$ are used. The values $\lambda_{d}\left(t, r_{v}\right)$ are obtained with the help of described method of the oscillating component $\tilde{z}$ quasi-envelope construction. This procedure is realized at each active interval $\tau_{a}$ of the limit cycle. In the regime of the dominant mode the values of the most differences $\Delta t_{m}[j]=\left\{t_{m}[l]-t_{m}[l-1]\right\}$ of the adjacent elements of the set $T_{m}=\{l\}$ are coincides approximately with semi-period $0,5 \tilde{\tau}_{j d}$ of the oscillating component that has the maximum amplitude.
After the operation of averaging $\tilde{\tau}_{d}=\frac{2}{L-1} \sum_{j=1}^{L-1} \Delta t_{m}[j]$, $L=\operatorname{dim} T_{m}$, the frequency of the dominant mode $\omega_{d}=2 \pi \tilde{\tau}_{d}^{-1}$ is defined. For identification of the dominant mode number " $d$ " the differences $\Delta \omega=\left|\omega_{j}-\omega_{d}\right| \quad(j=\overline{1, n})$ are investigated and it is accepted that $d=j$ from the condition $\Delta \omega_{j}=\left|\omega_{j}-\omega_{d}\right|=\min _{j}$. Further using the number " $d$ " of the dominant mode it is necessary to choose the corresponding influence function $\lambda_{d}\left(r_{v}\right)$. If $\lambda_{d}\left(r_{0}\right)>0$ it is required to select the new value of the parameter $r_{v}$ as it was written earlier. In the case when at $r_{v}=r_{0}$ the index $\lambda_{d}\left(r_{0}\right) \approx 0$ the intensity of the oscillating process $\tilde{x}(t)$ can be high or low (the amplitude of the dominant mode can be large or small). High intensity can lead to instability of the object motion. Because of this, intensity of the oscillating process is estimated by the mean value $\bar{z}_{m}$ of the set $Z_{m}$ elements. The mean value $\bar{z}_{m}$ is compared with the admissible value $\bar{z}_{m}^{*}$ and if $\bar{z}_{m}>\bar{z}_{m}^{*}$ the tuning (selection) of the parameter $r_{v}$ must be realized. If $\bar{z}_{m} \leq \bar{z}_{m}^{*}$ the tuning of the parameter $r_{v}=r_{0}$ is not required.

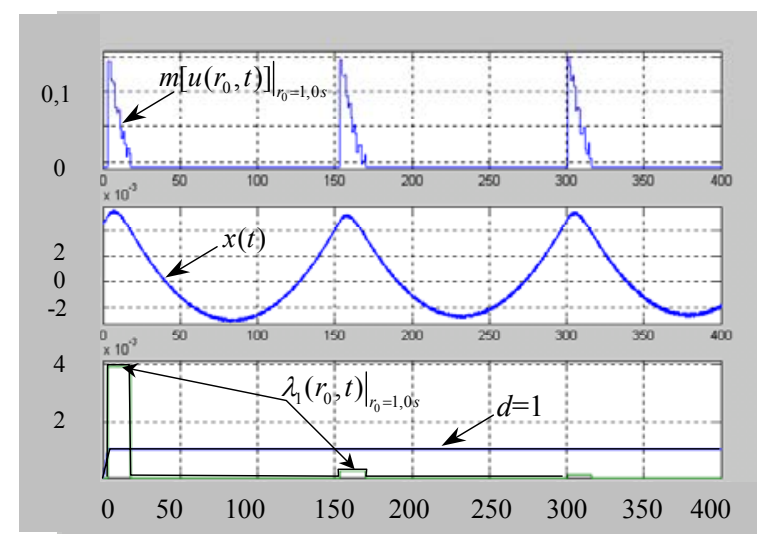

Fig. 2. Base regime of the LSS stabilization and outputs of the information module.

In Fig. 2 the example of the base control realization $(n=6)$ is shown.

Discrete analog of the PD-algorithm at $r_{0} \equiv T_{0}=1 \mathrm{~s}$ was used. It is clear that signals $\lambda_{1}(t)$ and $d(t)$ indicate the presence of the dominant mode $d(t)=1$ and its divergence $\left(\lambda_{1}(t)>0\right)$. Signals $\lambda_{1}(t), d(t)$ and $\bar{z}_{m}$ (signal $\bar{z}_{m}$ in Fig.2 is not depicted) were obtained by processing of the coordinate $z[k]$ on the active parts $\left(\tau_{a}=20 s\right)$ of the limit cycle. At $t>\tau_{a}$ the signals $\lambda_{d}(t), d(t)$ and $\bar{z}_{m}$ are retained fixed until the end of the current limit cycle. 


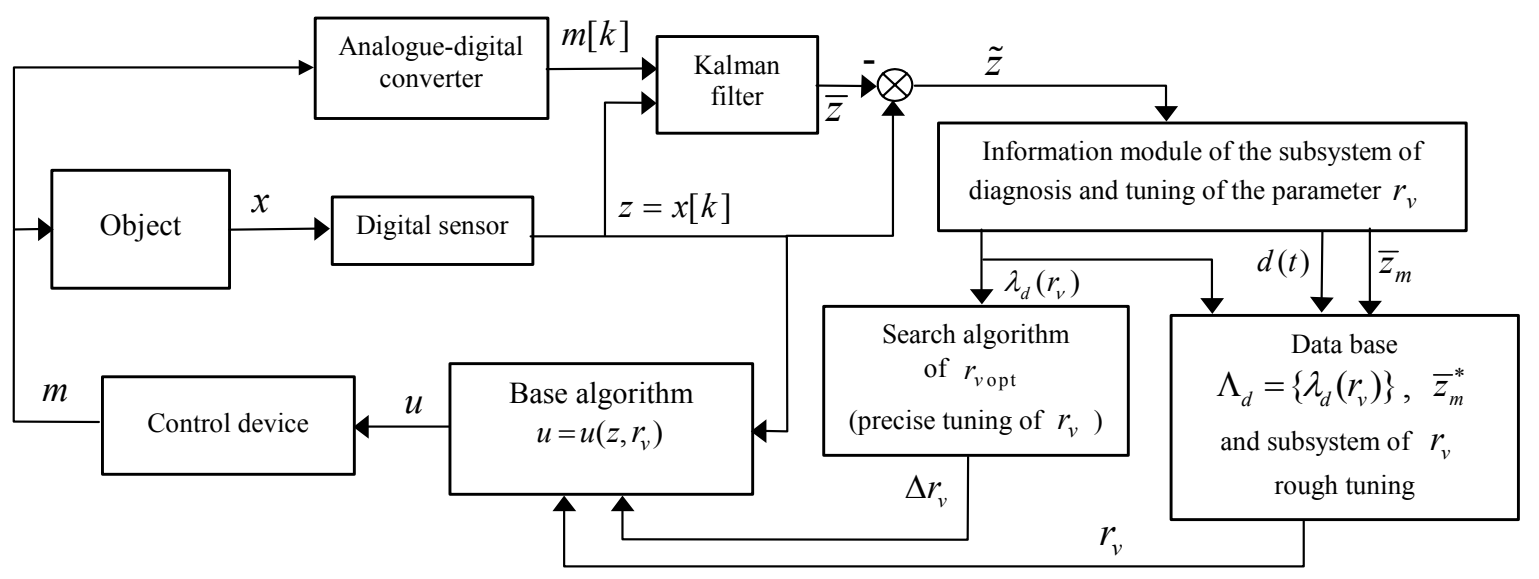

Fig. 3. Block scheme of the control system.

Described procedure of the parameter $r_{v}$ tuning is realized on-line using logical operations and the influence functions. This tuning is quick but rough. As a rule the new value $r_{1}$ is not equal to its optimal one $r_{1 \text { opt }}$ for that $\lambda_{d}\left(r_{1 \text { opt }}\right)=\lambda_{\text {min }} \forall r_{v} \in\left[r_{1}^{-}, r_{1}^{+}\right]$because the

influence functions are calculated in the process of the control system designing on the basis of designed parameters of the elastic oscillations. So it is appropriate to have an optimal subsystem for precise tuning of the parameter $r_{v}$.

For that one of the well-known algorithms of an extremum search (Krasovsky (ed.), 1987) may be used. In our case the minimum of the index $\lambda_{d}\left(r_{v}\right)$ in the domain $r_{v} \in\left[r_{1}^{-}, r_{1}^{+}\right]$is taken to be the extremum. In the optimal subsystem the test signal $\Delta r_{v}$ is generated and real index $\lambda_{d}\left(r_{v}\right)$ is calculated in the information module. The search is completed when $\lambda_{d}\left(r_{v}\right)=\lambda_{\min }$. After that the value $r_{v \text { opt }}$ is remained constant and the dominant mode amplitude is decreasing with maximum rate. But the amplitudes of other modes can increase as long as one of them will become as the dominant one. In this case the process of the parameter $r_{v}$ rough tuning is recurred.
By this means the suggested system has three loops. Its block-scheme is shown in Fig. 3.

The first, main, loop consists of the object, the analogue-digital converter, the control device and computer that realize the base algorithm. The main loop guarantees required quality of control at all values of the parameter $r_{v} \in\left[r_{\min }, r_{\max }\right]$ provided that the elastic oscillations $\tilde{x}(t)$ are small. Information module of the subsystem of diagnosis and tuning of the parameter $r_{v}$ estimates the index $\lambda_{d}$, number of the dominant mode " $d$ " and the index $\bar{z}_{m}$ using the elastic component $\tilde{z}[k]$. The second loop realizes the rough tuning of the parameter $r_{v}$ and the third one realizes the precise tuning of this parameter.

\section{COMPUTER SIMULATION OF THE SUGGESTED SYSTEM}

Suggested system was reproduced in MATLABSimulink taking into account nonlinear characteristics of the attitude sensor and control device. The values of the object parameters are depicted in the Table $1 \quad(n=6)$.

And it was assumed that the fundamental frequencies and coefficients of excitability can be given with the

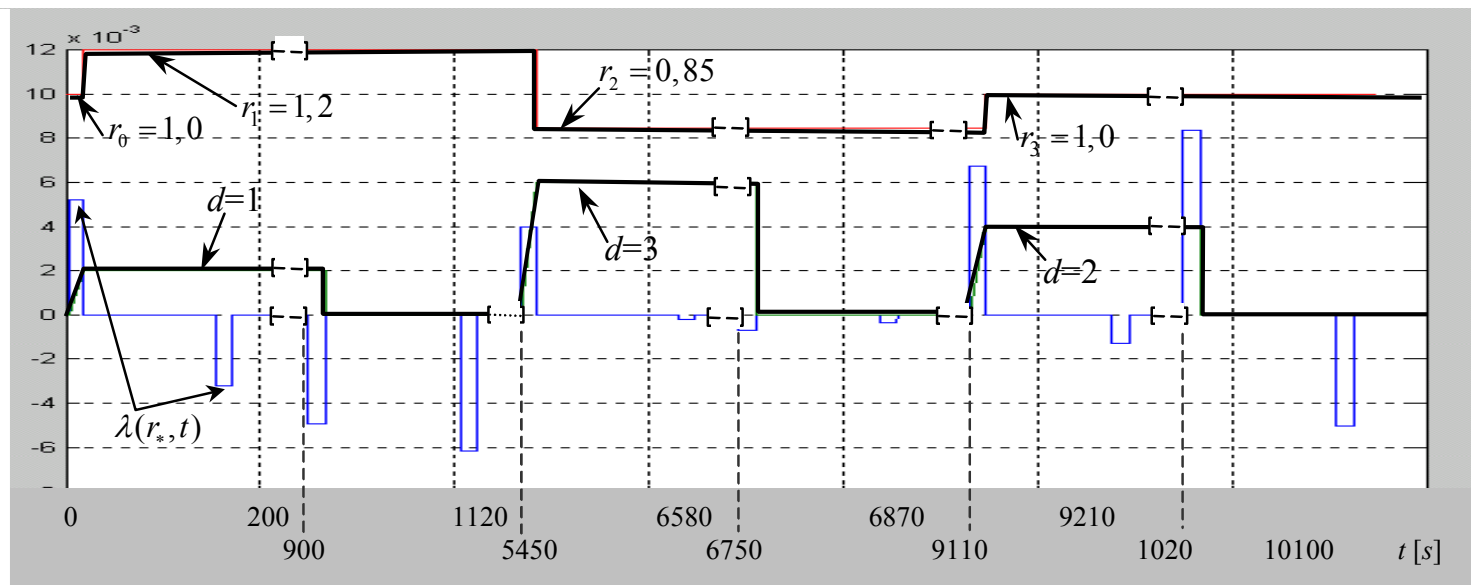

Fig. 4. Dynamics of the LSS stabilization process. 
errors (about $20 \%$ ). The discrete PD-algorithm (Krutova, 2001) with varying parameter $r_{v} \equiv T_{0}$ was chosen as the main algorithm of the stabilization. The initial conditions of the stabilization regime with respect to the «rigid» motion were: $\bar{x}_{0}=0,005 \mathrm{rad}, \dot{\bar{x}}_{0}=0$ and $T_{0}=1 \mathrm{~s}$. At that the first mode was as the dominant one. Other five modes have relatively small amplitudes.

In Fig. 4 some results of digital simulation are shown. Because of high duration of the stabilization process observation $\left(T_{\mathrm{obs}}=10300 \mathrm{~s}, \tau_{\Gamma}=150 \mathrm{~s}\right.$, $\left.\tau_{a}=20 \mathrm{~s}\right)$ the outputs $\lambda_{d}(t)$ and $d(t)$ are written only on typical parts of the process that are connected with changing in the condition of the LSS dynamics (for example, a change of the dominant mode number). The Fig. 4 shows that at the first active part of control it was defined out instability of the first mode $\lambda_{1}\left(T_{0}=1,0\right)=0,0052$ (Fig 1) and with regard to the signal $d=1$ and the influence function $\lambda_{1}\left(T_{0}\right)$ the parameter $T_{0}=1,0 \mathrm{~s}$ was changed to $T_{0}=1,2 \mathrm{~s}$ $\left(\lambda_{1}\left(T_{0}=1,2\right)=-0,0035<0\right.$ and it is the minimum value of $\lambda_{1}\left(T_{0}\right)$ ). So the first dominant mode became stable. At $t \geq 240 \mathrm{~s}$ the first mode amplitude is about zero. But the third mode became unstable (Fig 1) and from the instant $t \geq 545 \mathrm{~s}$ it was as the dominant mode that is $d=3$. This fact led to the next changing $T_{0}$ from $T_{0}=1,2 \mathrm{~s}$ to $T_{0}=0,8 \mathrm{~s}$ (Fig. 1). Thereafter the third mode began to converge. At $t \geq 6750 \mathrm{~s}$ its amplitude was about zero. Now the second mode was excited (the signal $d=2$ was appeared) and this led to the next changing $T_{0}$ from $T_{0}=0,8 \mathrm{~s}$ to $T_{0}=1,0 \mathrm{~s}$ (Fig 1 ) and to convergence of this dominant mode. Further these changes of the parameter $T_{0}$ are recurred during all duration of the LSS stabilization process. At other values of the object parameters the order of the dominant modes appearing will be different.

\section{CONCLUSION}

The results of this paper corroborate possibility of the high efficient control by large dimensional flexible mechanical systems in the case when it is not possible to choose the constant parameters of the base control algorithm which could guarantee the convergence of all elastic modes of the object construction. In suggested method the elements of the intellectual diagnosis are used for elastic oscillations damping. This damping is realized with the help of the adaptive tuning of the base algorithm parameter and without additional consumption of the energy for control. This method can be applied to many types of the space objects. But it should be noted that at the case when the frequencies of the elastic modes are close to the fundamental frequency of the "rigid" construction some troubles can occur.

\section{ACKNOWLEDGEMENT}

The work reported in the paper is a contribution to Project 03-01-00062, funded by Russian Foundation for Basic Research for which authors are grateful.

\section{REFERENCES}

Nurre G.S., R.S. Ryan, H.N. Skofield, J.I. Sims (1985). Dynamics and control of large space structures // Aerospace Technique. V. 3. № 6. C. 71-80.

Kirk, C. (Editor) (1990, 1993, 1996, 1999). Dynamics and control of structures in space. I-IV. Proceedings of the International conferences on $d y$ namics and control of structures in space, Computational mechanics publications, Southampton Boston.

Spencer B.F., T.T. Soong (1999). "New Applications and Development of Active, Semi-Active and Hybrid Control Techniques for Seismic and Non-Seismic Vibration in the Vibration in the USA", Proceedings of International Post SMIRT Conference on Seismic Isolation, Passive Energy Dissipation and active Control of Vibration of Structures, Korea.

Glumov V.M., S.D. Zemlyakov, V.Yu. Rutkovsky, V.M. Sukhanov (1998). Spatial Angular Motion of Flexible Spacecraft. The Modal Physical Model and its Characteristics. // Automation and Remote Control. Vol 59. N 12, Part 1. P. 17281738.

Krutova I.N., (2001). Studying Stability of the Flexible Spacecraft with a discrete Control System. Automation and Remote Control. Vol. 62, № 12. pp. 1964-1977.

Ermilova T.V., V.M. Sukhanov, A.S. Ermilov, V.G. Borisov (2004). Recurrent estimation of the angle motion coordinates of flexible objects of aerospace techniques. Aerospace Instrumentation. V. 6, P. 63-68. (In Russian).

Rutkovsky V.Yu., V.M. Sukhanov (1973). Specific relay control of flexible satellites. Proceedings of the 5-th IFAC Symposium on Automatic Control in Space, Genoa.

Dubrovin V., S. Subbotin, A. Boguslayev, V. Yatzenko (2003). Intelligent Means of Diagnostics and Prediction of Reliability of Air engines. Joint-Stock Company "Motor Sich", 2003.-279 p. (ISBN 966-7108-59-7). (In Russian).

Krasovsky A.A. (ed.) (1987). Reference book on the automatic control theory. M.: Nauka. (In Russian). 